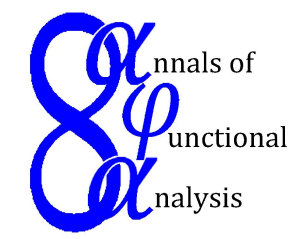

Ann. Funct. Anal. 6 (2015), no. 2, 26-32

http://doi.org/10.15352/afa/06-2-3

ISSN: 2008-8752 (electronic)

http://projecteuclid.org/afa

\title{
THE STABLE RANK OF $C^{*}$-MODULES
}

\author{
MAURICIO ACHIGAR
}

Communicated by T. Loring

\begin{abstract}
We prove equality between the Topological Stable Rank and the Bass Stable Rank for finitely generated projective left modules over a unital $C^{*}$-algebra. In order to do so, the concept of Stable Rank of a Hilbert module is introduced.
\end{abstract}

\section{INTRODUCTION AND PRELIMINARIES}

In the mid 1960s, H. Bass introduced the concept of Stable Rank of a ring $A$, now refered to as Bass Stable Rank and denoted by $\operatorname{Bsr}(A) \in \mathbb{N}$. In the late 1970s, R. B. Warfield extend this concept defining the Bass Stable Rank for modules over rings. Later, in [4], M. A. Rieffel introduced the notion of Topological Stable Rank for a Banach algebra $A, \operatorname{tsr}(A) \in \mathbb{N}$, as well as for Banach modules over unital Banach algebras. In this work, Rieffel shows that $\operatorname{Bsr}(A) \leq \operatorname{tsr}(A)$ holds for unital Banach Algebras and that $\operatorname{Bsr}(V) \leq \operatorname{tsr}(V)$ holds for finitely generated projective modules $V$ over unital $C^{*}$-algebras. In [3], R. H. Herman and L. N. Vaserstein prove that $\operatorname{Bsr}(A)=\operatorname{tsr}(A)$ for a unital $C^{*}$-algebra $A$.

In this article we show that $\operatorname{Bsr}(V)=\operatorname{tsr}(V)$ for finitely generated projective modules $V$ over unital $C^{*}$-algebras, using similar techniques to the ones presented in [3]. In order to generalize Herman-Vaserstein's theorem, we introduce the concept of Stable Rank for a Hilbert module. This definition is inspired by the works of Ara and Goodearl [1] and Blackadar [2].

Date: Received: Aug. 15, 2013; Revised: Mar. 11, 2014; Accepted: Apr. 20, 2014.

2010 Mathematics Subject Classification. Primary 46L08; Secondary 46L85, 46L05.

Key words and phrases. Bass stable rank, topological stable rank, $C^{*}$-algebra, $C^{*}$-module. 


\section{TYPES OF STABLE RANKS}

Definition 2.1. Let ${ }_{A} V$ be a left module over a ring $A$. The set of $n$-generators of $V$ is defined as

$$
\operatorname{Gen}_{n}(V)=\left\{\left(x_{1}, \ldots, x_{n}\right) \in V^{n}: A \cdot x_{1}+\cdots+A \cdot x_{n}=V\right\} .
$$

When $n=1$ we simply write $\operatorname{Gen}(V)=\operatorname{Gen}_{1}(V)$. We say that an $(n+1)$ generator $\left(x_{1}, \ldots, x_{n}, y\right) \in \operatorname{Gen}_{n+1}(V)$ is reducible if there exist $a_{1}, \ldots, a_{n} \in A$ such that $\left(x_{1}+a_{1} \cdot y, \ldots, x_{n}+a_{n} \cdot y\right) \in \operatorname{Gen}_{n}(V)$.

Remark 2.2. The column space $V^{n}$ can be viewed as a left module over the matrix ring $M_{n}(A)$. In that case we have

$$
\operatorname{Gen}_{n}\left({ }_{A} V\right)=\operatorname{Gen}\left({ }_{M_{n}(A)} V^{n}\right) .
$$

Definition 2.3. Let ${ }_{A} V$ be a left module over a ring $A$. The Bass Stable Rank of $V$, denoted $\operatorname{Bsr}(V)$, is defined as the least $n \in \mathbb{N}(n \geq 1)$ such that every $(n+1)$-generator $\left(x_{1}, \ldots, x_{n}, y\right) \in \operatorname{Gen}_{n+1}(V)$ is reducible.

When $V={ }_{A} A$ and $A$ has a unit, this definition becomes definition 2.1 in [4] and it's due to Bass. For arbitrary $V$ and unital $A$, the above definition is equivalent to definition [4, Definition 9.1] of Bass stable rank for modules, introduced by Warfield.

Definition 2.4. ([4, Definition 9.3]) Let ${ }_{A} V$ be a left Banach module over a Banach algebra $A$. The Topological Stable Rank of $V$ is defined as

$$
\operatorname{tsr}(V)=\min \left\{n \in \mathbb{N}: \operatorname{Gen}_{n}(V) \text { is dense in } V^{n}\right\} .
$$

\section{Stable Rank for Hilbert modules}

Definition 3.1. Given a right Hilbert module $X_{B}$ over a unital $C^{*}$-algebra $B$, we consider

$$
\mathrm{Um}_{n}(X)=\left\{\left(x_{1}, \ldots, x_{n}\right) \in X^{n}: \sum_{k}\left\langle x_{k}, x_{k}\right\rangle \in \mathrm{GL}(B)\right\} .
$$

An $n$-tuple in $\operatorname{Um}_{n}(X)$ is called unimodular tuple. If $n=1$ we write $\operatorname{Um}(X)=$ $\mathrm{Um}_{1}(X)$.

Remark 3.2. The column space $X^{n}$ can be viewed as a right $C^{*}$-module over $B$, and in this case we have

$$
\mathrm{Um}_{n}\left(X_{B}\right)=\mathrm{Um}\left(X_{B}^{n}\right) .
$$

Definition 3.3. Let $X_{B}$ be a right Hilbert module over a unital $C^{*}$-algebra $B$. We define the Stable Rank of $X_{B}$ as

$$
\operatorname{sr}\left(X_{B}\right)=\min \left\{n \in \mathbb{N}: \operatorname{Um}_{n}(X) \text { is dense in } X^{n}\right\} .
$$

Note that if $k \geq \operatorname{sr}\left(X_{B}\right)$ then $\operatorname{Um}_{k}(X)$ is dense in $X^{k}$.

Remark 3.4. For a unital $C^{*}$-algebra $B$, taking $X_{B}=B_{B}$ in the previous definition we recover Rieffel's definition of (left) topological stable rank of $B$ [4, Definition 1.4]. Indeed, Lemma 3.7 or Remark 3.8 can be used to see that $\operatorname{Um}_{n}\left(B_{B}\right)=$ $\operatorname{Lg}_{n}(B)$, the later being the set of left $n$-generators of $B$ considered in [4]. Then, $\operatorname{sr}\left(B_{B}\right)=\operatorname{tsr}(B)(=\operatorname{Bsr}(B))$. 
Remark 3.5. For projections $p, q$ in a $C^{*}$-algebra $A$, Blackadar ([2]) considered the set

$$
\operatorname{Lg}_{(p, q)}(A)=\{x \in p A q: \exists y \in q A p \text { such that } y x=q\}
$$

and used the condition of $\operatorname{Lg}_{(p, q)}(A)$ being dense in $p A q$. Taking $X$ as the skew corner $X=p A q$ and $B=q A q$ we have, by Lemma 3.7, that $\operatorname{Lg}_{(p, q)}(A)=\operatorname{Um}\left(X_{B}\right)$, and $\operatorname{Lg}_{(p, q)}(A)$ is dense in $p A q$ if and only if $\operatorname{sr}\left(X_{B}\right)=1$.

Example 3.6. Let $A$ be a unital $C^{*}$-algebra and consider the set $M_{n \times m}(A)$ as a right $C^{*}$-module over $M_{m}(A)$ with formal matrix operations. The stable rank of $M_{n \times m}(A)$ is

$$
\operatorname{sr}\left(M_{n \times m}(A)\right)=\left\lceil\frac{\operatorname{sr}(A)+m-1}{n}\right\rceil .
$$

This expression extends the well-known formula for $\operatorname{sr}\left(M_{n}(A)\right)([4$, theorem 6.1$])$.

Proof. Firstly, by Lemma 3.7 we know that $\operatorname{Um}\left(M_{n \times m}(A)\right)$ is the set of left invertible $n \times m$ matrices over $A$. Then, by [2, Corollary 4.3] we have

$\operatorname{Um}\left(M_{(n+1) \times 1}(A)\right)$ is dense iff $\operatorname{Um}\left(M_{(n+k) \times k}(A)\right)$ is dense,

where "Um $\left(M_{r \times s}(A)\right)$ dense" means dense in $M_{r \times s}(A)$. Equivalently,

$\mathrm{Um}\left(M_{r \times s}(A)\right)$ is dense iff $\operatorname{Um}\left(M_{(r-s+1) \times 1}(A)\right)$ is dense, for $r \geq s$.

Secondly, we can realize $M_{n \times m}(A)$ as a skew corner of $M_{\bar{n}}(A)$ for $\bar{n}$ large in the following way: $M_{n \times m}(A) \cong p M_{\bar{n}}(A) q$ for $p, q \in M_{\bar{n}}(A)$ diagonal projections of ranks $n$ and $m$, respectively. Then, by [2, Proposition 3.2.iii] we have

$$
\text { If } \operatorname{Um}\left(M_{n \times m}(A)\right) \text { is dense then } n \geq m \text {. }
$$

For $k \in \mathbb{N}$, we have $k \geq \operatorname{sr}\left(M_{n \times m}(A)\right)$ iff $\operatorname{Um}_{k}\left(M_{n \times m}(A)\right)$ is dense. Identifying the column space $M_{n \times m}(A)^{k}$ with $M_{n k \times m}(A)$ we have $\operatorname{Um}_{k}\left(M_{n \times m}(A)\right)=$ $\operatorname{Um}\left(M_{n \times m}(A)^{k}\right)=\operatorname{Um}\left(M_{n k \times m}(A)\right)$. If $\operatorname{Um}\left(M_{n k \times m}(A)\right)$ is dense then $n k \geq m$, by (3.3), and $\operatorname{Um}\left(M_{(n k-m+1) \times 1}(A)\right)$ is dense, by (3.2). Therefore $n k-m+1 \geq \operatorname{sr}(A)$ by definition of $\operatorname{sr}(A)$, and then $k \geq \frac{\operatorname{sr}(A)+m-1}{n}$. Conversely, the inequalities imply $n k \geq m$ and the density of $\operatorname{Um}\left(M_{(n k-m+1) \times 1}(A)\right)$, then by (3.2) $\operatorname{Um}\left(M_{n k \times m}(A)\right)$ $\left(=\operatorname{Um}_{k}\left(M_{n \times m}(A)\right)\right)$ is dense, and finally $k \geq \operatorname{sr}\left(M_{n \times m}(A)\right)$. Thus we have shown that $k \geq \operatorname{sr}\left(M_{n \times m}(A)\right)$ iff $k \geq \frac{\operatorname{sr}(A)+m-1}{n}$, which is equivalent to (3.1).

Lemma 3.7. Let $X_{B}$ be a unital Hilbert module. For $x \in X$, the following are equivalent:

(a) $\langle x, x\rangle \in G L(B)$.

(b) There exists $y \in X$ such that $\langle y, x\rangle=1$.

(b*) There exists $y \in X$ such that $\langle x, y\rangle=1$.

(c) There exists $y \in X$ such that $\langle y, x\rangle \in G L(B)$.

(c*) There exists $y \in X$ such that $\langle x, y\rangle \in G L(B)$.

Proof. It suffices to show that (a) is equivalent to (b). If $\langle x, x\rangle \in \mathrm{GL}(B)$ let $b=\langle x, x\rangle$ and $y=x\left(b^{-1}\right)^{*}$. Then $\langle y, x\rangle=b^{-1}\langle x, x\rangle=1$. On the other hand, if $\langle y, x\rangle=1$ we have $1=\langle y, x\rangle^{*}\langle y, x\rangle \leq\|y\|^{2}\langle x, x\rangle$, so that $\langle x, x\rangle \in \operatorname{GL}(B)$. 
Remark 3.8. Applying Lemma 3.7 to $X^{n}$ we obtain different expressions for $\mathrm{Um}_{n}(X)$. For example, using (b) we have

$$
\operatorname{Um}_{n}(X)=\left\{\left(x_{1}, \ldots, x_{n}\right) \in X^{n}: \exists y_{1}, \ldots, y_{n} \in X / \sum_{k}\left\langle y_{k}, x_{k}\right\rangle=1\right\}
$$

Lemma 3.9. Let $X_{B}$ be a unital Hilbert module. Then $X_{B}$ is full if and only if there exists $n \in \mathbb{N}$ such that $U_{n}(X) \neq \varnothing$.

Proof. The module $X_{B}$ is full if and only if the $*$-ideal $J=\operatorname{span}(\langle X, X\rangle)$ is dense in $B$, iff $J \cap \operatorname{GL}(B) \neq \varnothing$, iff $1 \in J(=B)$, iff there exists $n \in \mathbb{N}$ and $x_{1}, \ldots, x_{n}, y_{1}, \ldots, y_{n} \in X$ such that $\sum_{k}\left\langle x_{k}, y_{k}\right\rangle=1$, iff there exists $n \in \mathbb{N}$ and $x, y \in X^{n}$ such that $\langle x, y\rangle=1$, iff there exists $n \in \mathbb{N}$ such that $\operatorname{Um}_{n}(X) \neq \varnothing$.

Notice that if $X_{B}$ is not full then $\operatorname{Um}_{n}(X)=\varnothing$ for all $n \in \mathbb{N}$, and therefore $\operatorname{sr}(X)=\infty$. Thus, throughout this paper we shall consider $X_{B}$ to be full.

Definition 3.10. A $C^{*}$-correspondence is a right Hilbert module $X_{B}$ equipped with a left action $A \rightarrow \mathcal{L}\left(X_{B}\right)$ of a $C^{*}$-algebra $A$ by adjointable operators. When $B$ has a unit we say that the correspondence is right-unital.

Lemma 3.11. Let ${ }_{A} X_{B}$ be a full and right-unital $C^{*}$-correspondence. Then

$$
\operatorname{Gen}_{n}\left({ }_{A} X\right) \subseteq U m_{n}\left(X_{B}\right) \quad \forall n \in \mathbb{N} .
$$

Proof. Since $\operatorname{Gen}_{n}\left({ }_{A} X\right)=\operatorname{Gen}\left(M_{n}(A) X^{n}\right), \operatorname{Um}_{n}\left(X_{B}\right)=\operatorname{Um}\left(X_{B}^{n}\right)$, and the $C^{*}$ correspondence $M_{n}(A) X_{B}^{n}$ is full and right-unital, we may assume $n=1$. As $X_{B}$ is full there exist $x_{1}, \ldots, x_{r}, y_{1}, \ldots, y_{r} \in X$ such that $\sum_{k}\left\langle x_{k}, y_{k}\right\rangle=1$. If $x \in \operatorname{Gen}\left({ }_{A} X\right)$ let $a_{1}, \ldots, a_{r} \in A$ be such that $x_{k}=a_{k} \cdot x$, for $k=1, \ldots, r$. Then,

$$
1=\sum_{k}\left\langle x_{k}, y_{k}\right\rangle=\sum_{k}\left\langle a_{k} \cdot x, y_{k}\right\rangle=\sum_{k}\left\langle x, a_{k}^{*} \cdot y_{k}\right\rangle=\left\langle x, \sum_{k} a_{k}^{*} \cdot y_{k}\right\rangle
$$

so $x \in \operatorname{Um}\left(X_{B}\right)$.

Definition 3.12. A vector space $X$ is said to be a $C^{*}$-bimodule whenever it is equipped with compatible left and right Hilbert module structures (that is $x \cdot\langle y, z\rangle_{R}=\langle x, y\rangle_{L} \cdot z$, for $\left.x, y, z \in X\right)$ over $C^{*}$-algebras $A$ and $B$, respectively.

Lemma 3.13. Let ${ }_{A} X_{B}$ be a right-full and right-unital $C^{*}$-bimodule. Then

$$
U m_{n}\left(X_{B}\right) \subseteq \operatorname{Gen}_{n}\left({ }_{A} X\right) \quad \forall n \in \mathbb{N} .
$$

Proof. Since $\operatorname{Gen}_{n}\left({ }_{A} X\right)=\operatorname{Gen}\left(M_{n}(A) X^{n}\right), \operatorname{Um}_{n}\left(X_{B}\right)=\operatorname{Um}\left(X_{B}^{n}\right)$ and the $C^{*}$ bimodule $M_{n}(A) X_{B}^{n}$ is right-full and right-unital, we may assume $n=1$. Given $x \in \operatorname{Um}(X)$, let $y \in X$ be such that $\langle y, x\rangle_{R}=1$. Then, for all $z \in X$ we have $z=z \cdot 1=z \cdot\langle y, x\rangle_{R}=\langle z, y\rangle_{L} \cdot x$. Then $x \in \operatorname{Gen}(X)$.

Proposition 3.14. Let ${ }_{A} X_{B}$ be a right-full and right-unital $C^{*}$-bimodule. Then

$$
U m_{n}\left(X_{B}\right)=\operatorname{Gen}_{n}\left({ }_{A} X\right) \quad \forall n \in \mathbb{N} \quad \text { and } \quad \operatorname{tsr}\left({ }_{A} X\right)=\operatorname{sr}\left(X_{B}\right) .
$$




\section{Stable RANK Inequality For $C^{*}$-MOdules}

[4, Proposition 9.7] says that $\operatorname{Bsr}(V) \leq \operatorname{tsr}(V)$ for a finitely generated projective left module ${ }_{A} V$ over a unital $C^{*}$-algebra $A$. Inspired by this, we prove a similar result in the $C^{*}$-module context, namely, if $X$ is a right-full and right-unital $C^{*}$-bimodule, then $\operatorname{Bsr}(X) \leq \operatorname{tsr}(X)$.

Lemma 4.1. (Warfield Condition, [4, Propositions 2.2 and 9.2]) Let ${ }_{A} X_{B}$ be a right-full and right-unital $C^{*}$-bimodule and $x_{1}, \ldots, x_{n+1} \in X$. The following conditions are equivalent:

(a) $\exists a_{1}, \ldots, a_{n} \in A /\left(x_{1}+a_{1} \cdot x_{n+1}, \ldots, x_{n}+a_{n} \cdot x_{n+1}\right) \in U m_{n}(X)$.

(b) $\exists y_{1}, \ldots, y_{n+1} \in X / \sum_{k=1}^{n+1}\left\langle y_{k}, x_{k}\right\rangle_{R}=1$ and $\left(y_{1}, \ldots, y_{n}\right) \in U m_{n}(X)$.

Proof. If $\left(x_{1}+a_{1} \cdot x_{n+1}, \ldots, x_{n}+a_{n} \cdot x_{n+1}\right) \in \mathrm{Um}_{n}(X)$, there exist $y_{1}, \ldots, y_{n} \in X$ such that $\sum_{k=1}^{n}\left\langle y_{k}, x_{k}+a_{k} \cdot x_{n+1}\right\rangle_{R}=1$. Then we have $\left(y_{1}, \ldots, y_{n}\right) \in \operatorname{Um}_{n}(X)$ by Lemma 3.7, and

$$
\sum_{k=1}^{n}\left\langle y_{k}, x_{k}\right\rangle_{R}+\left\langle\sum_{k=1}^{n} a_{k}^{*} \cdot y_{k}, x_{n+1}\right\rangle_{R}=1
$$

Taking $y_{n+1}=\sum_{k=1}^{n} a_{k}^{*} \cdot y_{k}$ we obtain (b).

Conversely, if condition (b) holds, as $\left(y_{1}, \ldots, y_{n}\right) \in \operatorname{Um}_{n}(X)$ there are $z_{1}, \ldots, z_{n} \in$ $X$ such that $\sum_{k=1}^{n}\left\langle y_{k}, z_{k}\right\rangle_{R}=1$. Let $a_{k}=\left\langle z_{k}, y_{n+1}\right\rangle_{L}$, for $k=1, \ldots, n$. Then

$$
\sum_{k=1}^{n}\left\langle y_{k}, x_{k}+a_{k} \cdot x_{n+1}\right\rangle_{R}=\sum_{k=1}^{n}\left\langle y_{k}, x_{k}\right\rangle_{R}+\left\langle\sum_{k=1}^{n} a_{k}^{*} \cdot y_{k}, x_{n+1}\right\rangle_{R}
$$

Now, we have

$$
\begin{array}{r}
\sum_{k=1}^{n} a_{k}^{*} \cdot y_{k}=\sum_{k=1}^{n}\left\langle y_{n+1}, z_{k}\right\rangle_{L} \cdot y_{k}=\sum_{k=1}^{n} y_{n+1} \cdot\left\langle z_{k}, y_{k}\right\rangle_{R} \\
=y_{n+1} \cdot \sum_{k=1}^{n}\left\langle z_{k}, y_{k}\right\rangle_{R}=y_{n+1} \cdot 1=y_{n+1} .
\end{array}
$$

Then, the right-hand side of equation (4.1) equals 1 by (b), and (a) holds.

The proof of the following proposition is analogous to that of [4, Theorem 2.3].

Proposition 4.2. ([4, Proposition 9.7]) Let ${ }_{A} X_{B}$ be a right-full and right-unital $C^{*}$-bimodule. Then

$$
\operatorname{Bsr}(X) \leq \operatorname{tsr}(X)
$$

Proof. Let $n=\operatorname{tsr}(X)=\operatorname{sr}(X)$. Given $\left(x_{1}, \ldots, x_{n+1}\right) \in \operatorname{Gen}_{n+1}\left({ }_{A} X\right)=\operatorname{Um}_{n+1}\left(X_{B}\right)$ consider $z_{1}, \ldots, z_{n+1} \in X$ such that $\sum_{k=1}^{n+1}\left\langle z_{k}, x_{k}\right\rangle_{R}=1$. For $k=1, \ldots, n$, pick perturbations $\bar{z}_{k} \simeq z_{k}$ with $\left(\bar{z}_{1} \ldots, \bar{z}_{n}\right) \in \operatorname{Um}_{n}(X)$ so that

$$
d^{*}:=\left\langle\bar{z}_{1}, x_{1}\right\rangle_{R}+\cdots+\left\langle\bar{z}_{n}, x_{n}\right\rangle_{R}+\left\langle z_{n+1}, x_{n+1}\right\rangle_{R} \in \mathrm{GL}(B) .
$$


Then, taking $y_{1}=\bar{z}_{1} \cdot d^{-1}, \ldots, y_{n}=\bar{z}_{n} \cdot d^{-1}, y_{n+1}=z_{n+1} \cdot d^{-1}$ we have $\left(y_{1}, \ldots, y_{n}\right)=$ $\left(\bar{z}_{1}, \ldots, \bar{z}_{n}\right) \cdot d^{-1} \in \mathrm{Um}_{n}(X)$ and $\sum_{k=1}^{n+1}\left\langle y_{k}, x_{k}\right\rangle_{R}=1$. By the previous lemma $\left(x_{1}, \ldots, x_{n+1}\right)$ is reducible, and then $\operatorname{Bsr}(X) \leq n$.

\section{Herman-VASERstien theOREM FOR $C^{*}$-MOdUles}

Herman-Vaserstein theorem states that for a unital $C^{*}$-algebra $A, \operatorname{tsr}(A) \leq$ $\operatorname{Bsr}(A)$. In this section we obtain $\operatorname{tsr}(X) \leq \operatorname{Bsr}(X)$ for a right-full and rightunital Hilbert bimodule $X$.

Lemma 5.1. Let $X_{B}$ be a full and unital Hilbert module. Given $x_{1}, \ldots, x_{n}$, $u_{1}, \ldots, u_{r} \in X$ such that $\sum_{k}\left\langle u_{k}, u_{k}\right\rangle=1$, and $\varepsilon>0$, let $b_{0}=\sum_{i}\left\langle x_{i}, x_{i}\right\rangle, b=(1-$ $\left.\frac{b_{0}}{\varepsilon}\right)^{+}$and $y_{k}=u_{k} \cdot b$, for $k=1, \ldots, r$. Then $\left(x_{1}, \ldots, x_{n}, y_{1}, \ldots, y_{r}\right) \in U m_{n+r}\left(X_{B}\right)$.

Proof. Let $x=\left(x_{1}, \ldots, x_{n}\right), u=\left(u_{1}, \ldots, u_{r}\right)$ and $y=\left(y_{1}, \ldots, y_{r}\right)$, then $y=u \cdot b$ and $b_{0}=\langle x, x\rangle$. Consider the commutative $C^{*}$-subalgebra $B_{0}:=C^{*}\left(1, b_{0}\right) \subseteq B$. Let $c \in B$ the element given by

$$
c=\langle y, y\rangle=\langle u \cdot b, u \cdot b\rangle=b^{*}\langle u, u\rangle b=b^{*} b=\left[\left(1-\frac{b_{0}}{\varepsilon}\right)^{+}\right]^{2} .
$$

Consequently $c$ and $b_{0}$ belong to $B_{0}^{+}$and do not have common roots. Therefore

$$
\langle(x, y),(x, y)\rangle=\langle x, x\rangle+\langle y, y\rangle=b_{0}+c \in \mathrm{GL}\left(B_{0}\right) \subseteq \mathrm{GL}(B) .
$$

That is, $(x, y)=\left(x_{1}, \ldots, x_{n}, y_{1}, \ldots, y_{r}\right) \in \operatorname{Um}_{n+r}\left(X_{B}\right)$.

Theorem 5.2. Let ${ }_{A} X_{B}$ be a right-full and right-unital $C^{*}$-bimodule. Then

$$
\operatorname{Bsr}(X)=\operatorname{tsr}(X) \text {. }
$$

Proof. By Proposition 4.2 it suffices to show $\operatorname{Bsr}(X) \geq \operatorname{tsr}(X)$. Suppose $\operatorname{Bsr}\left({ }_{A} X\right)=$ $n$ and let $x=\left(x_{1}, \ldots, x_{n}\right) \in X^{n}, \varepsilon>0$ be given. As $X$ is right-full and rightunital, by Lemma 3.9, there exists $u=\left(u_{1}, \ldots, u_{r}\right) \in \operatorname{Um}_{r}(X)$ for suitable $r \in \mathbb{N}$. Replacing $u$ with $u \cdot\langle u, u\rangle_{R}^{-1 / 2}$, we may suppose $\langle u, u\rangle_{R}=1$. Taking $b_{0}, b$ and $y$ as in Lemma 5.1 we have that $(x, y) \in \operatorname{Um}_{n+r}\left(X_{B}\right)=\operatorname{Gen}_{n+r}\left({ }_{A} X\right)$. Then, as $\operatorname{Bsr}\left({ }_{A} X\right)=n$, the generator $(x, y)$ can be reduced $r$ times to an $n$-generator. Therefore, there exists $a \in M_{n \times r}(A)$ such that $x+a \cdot y \in \operatorname{Gen}_{n}\left({ }_{A} X\right)=\operatorname{Um}_{n}\left(X_{B}\right)$. Let

$$
\begin{aligned}
& k>\frac{\|a\|}{\varepsilon}, \quad d=1+k b \in B^{+} \cap \operatorname{GL}(B) \quad \text { and } \\
& x^{\prime}=(x+a \cdot y) \cdot d^{-1} \in \operatorname{Um}_{n}(X)=\operatorname{Gen}_{n}(X),
\end{aligned}
$$

where $\|a\|$ is the norm of $a$ as a $B$-adjointable operator $a: X^{r} \rightarrow X^{n}$.

We have $x-x^{\prime}=(x \cdot d-x-a \cdot y) \cdot d^{-1}=(x \cdot k b-a \cdot y) \cdot d^{-1}$ and

$$
\left\|x-x^{\prime}\right\| \leq\left\|x \cdot k b d^{-1}\right\|+\left\|a \cdot y \cdot d^{-1}\right\| .
$$

As $b_{0}=\langle x, x\rangle_{R}$, we have

$$
\left|x \cdot k b d^{-1}\right|_{R}^{2}=\left(k b d^{-1}\right)^{*}\langle x, x\rangle_{R}\left(k b d^{-1}\right)=\left(k b d^{-1}\right)^{*} b_{0}\left(k b d^{-1}\right) .
$$

Now, as $b=\left(1-\frac{b_{0}}{\varepsilon}\right)^{+}$, we have $d=1+k b \in C^{*}\left(1, b_{0}\right) \cong C(T)$ which is commutative. Therefore $k b d^{-1}=k b(1+k b)^{-1}=b\left(\frac{1}{k}+b\right)^{-1} \leq 1$ in $C(T)$ and consequently 
$\left(k b d^{-1}\right)^{*} b_{0}\left(k b d^{-1}\right) \leq b_{0}$. Moreover, if $b_{0}(t)>\varepsilon$ for suitable $t \in T$, then $b(t)=0$, because $b=\left(1-\frac{b_{0}}{\varepsilon}\right)^{+}$. Hence $\left(k b d^{-1}\right)^{*} b_{0}\left(k b d^{-1}\right) \leq \varepsilon$ and

$$
\left\|x \cdot k b d^{-1}\right\|=\left\|\left|x \cdot k b d^{-1}\right|_{R}^{2}\right\|^{1 / 2} \leq \sqrt{\varepsilon}
$$

On the other hand, since $y=u \cdot b$ we have

$$
\left\|a \cdot y \cdot d^{-1}\right\|=\left\|\frac{a}{k} \cdot u \cdot k b d^{-1}\right\| \leq \frac{\|a\|}{k}\|u\|\left\|k b d^{-1}\right\|<\varepsilon,
$$

where we have used that $\|a\| / k<\varepsilon,\|u\|=1$ and $\left\|k b d^{-1}\right\| \leq 1$.

Thus we can estimate (5.1) using equations (5.2) and (5.3) to get

$$
\left\|x-x^{\prime}\right\|<\sqrt{\varepsilon}+\varepsilon .
$$

Then, $x^{\prime}$ can be taken arbitrarily close to $x$ and $x^{\prime} \in \operatorname{Gen}_{n}(X)$. Therefore, $\operatorname{Gen}_{n}(X)$ is dense and $\operatorname{tsr}(X) \leq n$.

Remark 5.3. If ${ }_{A} X$ is a finitely generated projective module over a unital $C^{*}$ algebra $A$ we can make it into a right-full and right-unital $C^{*}$-bimodule in the following way. The module ${ }_{A} X$ is a direct summand of $A^{n}$ for suitable $n \in$ $\mathbb{N}$, and is therefore the range of a (selfadjoint) projection $p \in M_{n}(A)$. Then we have ${ }_{A} X$ as the submodule ${ }_{A}\left(A^{n} p\right)$ of ${ }_{A} A^{n}$. As we actually have a Hilbert $A-M_{n}(A)$ bimodule structrue on $A^{n}$ (thinking of $A^{n}$ as a row space and using the usual matrix operations) we obtain, by restriction, an $A-p M_{n}(A) p C^{*}$-bimodule ${ }_{A}\left(A^{n} p\right)_{p M_{n}(A) p}$, which is right-full and right-unital.

Combining this construction with Theorem 5.2 we have that $\operatorname{Bsr}(X)=\operatorname{tsr}(X)$ for every finitely generated projective left module over a unital $C^{*}$-algebra.

Acknowledgement. The author wishes to thank his friend Janine Bachrachas for her help editing this article.

Partially supported by Proyecto Fondo Clemente Estable FCE2007_731.

\section{REFERENCES}

1. P. Ara and K.R. Goodearl, Stable rank of corner rings, Proc. Amer. Math. Soc. 133 (2005), 370-386.

2. B. Blackadar, The stable rank of full corners in $C^{*}$-algebras, Proc. Amer. Math. Soc. 312 (2004), 2945-2950.

3. R.H. Herman and L.N. Vaserstein, The stable range of $C^{*}$-algebras, Invent. Math. 77 (1984), 553-555.

4. M.A. Rieffel, Dimension and stable rank in the K-theory of $C^{*}$-algebras, Proc. London Math. Soc. 46 (1983), 301-333.

Facultad de Ciencias, Iguá 4225, CP 11400, Montevideo, Uruguay.

E-mail address: achigar@cmat.edu.uy; mauricio.achigar3@gmail.com 